TRANSACTIONS OF THE

AMERICAN MATHEMATICAL SOCIETY

Volume 365, Number 2, February 2013, Pages 579-590

S 0002-9947(2012)05556-5

Article electronically published on September 19, 2012

\title{
GOOD REDUCTION OF THE BRAUER-MANIN OBSTRUCTION
}

\author{
JEAN-LOUIS COLLIOT-THÉLÈNE AND ALEXEI N. SKOROBOGATOV \\ Abstract. For a smooth and projective variety over a number field with \\ torsion-free geometric Picard group and finite transcendental Brauer group \\ we show that only the archimedean places, the primes of bad reduction and \\ the primes dividing the order of the transcendental Brauer group can turn up \\ in the description of the Brauer-Manin set.
}

\section{INTRODUCTION}

We assume that the reader is familiar with the basic theory of the Brauer group 8 and of the Brauer-Manin obstruction; see [3, §3.1] or [16, §5.2].

Let us briefly recall some notation. To any scheme $X$ one associates its (Grothendieck) Brauer group $\operatorname{Br}(X)=\mathrm{H}_{\text {ét }}^{2}\left(X, \mathbb{G}_{m}\right)$. Let $k$ be a field, $\bar{k}$ be an algebraic closure of $k, \Gamma=\operatorname{Gal}(\bar{k} / k)$. If $X$ is a variety over $k$ we write $\bar{X}=X \times_{k} \bar{k}$. Recall the standard notation

$$
\operatorname{Br}_{0}(X)=\operatorname{Im}[\operatorname{Br}(k) \rightarrow \operatorname{Br}(X)], \quad \operatorname{Br}_{1}(X)=\operatorname{Ker}[\operatorname{Br}(X) \rightarrow \operatorname{Br}(\bar{X})] .
$$

The group $\operatorname{Br}_{1}(X)$ is called the algebraic Brauer group of $X$, and the quotient $\operatorname{Br}(X) / \operatorname{Br}_{1}(X)$ is sometimes called the transcendental Brauer group of $X$, a terminology we adopt in this note.

Let $k$ be a number field, let $k_{v}$ be the completion of $k$ at a place $v$, and let $\mathbb{A}_{k}$ be the ring of adèles of $k$, i.e. the restricted product of $k_{v}$ for all places $v$ of $k$, taken with respect to the rings of integers $\mathcal{O}_{v} \subset k_{v}$. Let $\mathcal{O}$ be the ring of integers of $k$. Given a finite set $S$ of places of $k$, we let $\mathcal{O}_{S}$ be the subring of $k$ consisting of the elements that are integral at the primes not in $S$.

Given a variety $X$ over $k$, the set $X\left(\mathbb{A}_{k}\right)^{\mathrm{Br}} \subset X\left(\mathbb{A}_{k}\right)$ is the set of adèles of $X$ which are orthogonal to the Brauer group of $X$ with respect to the Brauer-Manin pairing.

Our aim is to explore what can be proved in the direction of the following question.

Question 1. Let $k$ be a number field and let $S$ be a finite set of places containing the archimedean places. Let $\mathcal{X}$ be a smooth projective $\mathcal{O}_{S}$-scheme with geometrically integral fibres. Let $X / k$ be its generic fibre. Assume that $\operatorname{Pic}(\bar{X})$ is a finitely generated torsion-free abelian group. Does there exist an open and closed set $Z \subset \prod_{v \in S} X\left(k_{v}\right)$ such that

$$
X\left(\mathbb{A}_{k}\right)^{\mathrm{Br}}=Z \times \prod_{v \notin S} X\left(k_{v}\right) ?
$$

Received by the editors September 1, 2010.

2010 Mathematics Subject Classification. Primary 14F22, 14G05, 11G35, 11G25.

(C)2012 American Mathematical Society Reverts to public domain 28 years from publication 
The assumption on the Picard group is equivalent to the conjunction of two geometric hypotheses: the coherent cohomology group $\mathrm{H}^{1}\left(X, O_{X}\right)$ is zero, and the geometric Néron-Severi group $\operatorname{NS}(\bar{X})$ is torsion-free.

Peter Swinnerton-Dyer asked us this question in connection with his recent work 19. Theorem 3.1 below gives an affirmative answer under the additional hypothesis that the transcendental Brauer group of $X$ (as defined above) is finite, and $S$ contains all the primes dividing its order. A criterion that uses only a geometric hypothesis on $\bar{X}$ is given in Corollary 3.2 For example, the answer is positive whenever $X$ is a surface of geometric genus 0. In Corollary 3.3 , using the results of 9], we give a positive answer to Question 1 for arbitrary smooth diagonal quartic surfaces over $\mathbb{Q}$, a case which is not covered by Corollary 3.2. For general varieties, Proposition 4.1 relates the finiteness property of the transcendental Brauer group to the Tate conjecture for divisors.

\section{Preliminaries}

Given an abelian group $A$, a positive integer $n$ and a prime number $\ell$, we denote by $A[n] \subset A$ the group of elements annihilated by $n$, and by $A\{\ell\} \subset A$ the group of elements annihilated by some power of $\ell$.

The following lemma is well known.

Lemma 1.1. Let $X$ be a smooth, projective and geometrically integral variety over a number field $k$ such that $\operatorname{Pic}(\bar{X})$ is a finitely generated torsion-free abelian group, and $\operatorname{Br}(X) / \operatorname{Br}_{1}(X)$ is finite. Then $X\left(\mathbb{A}_{k}\right)^{\mathrm{Br}}$ is open and closed in $X\left(\mathbb{A}_{k}\right)$.

Proof. From the spectral sequence

$$
E_{2}^{p q}=\mathrm{H}^{p}\left(k, \mathrm{H}_{\text {ét }}^{q}\left(\bar{X}, \mathbb{G}_{m}\right)\right) \Rightarrow \mathrm{H}_{\text {ét }}^{p+q}\left(X, \mathbb{G}_{m}\right)
$$

we see that the quotient $\operatorname{Br}_{1}(X) / \operatorname{Br}_{0}(X)$ is a subgroup of $\mathrm{H}^{1}(k, \operatorname{Pic}(\bar{X}))$, and this last group is finite since $\operatorname{Pic}(\bar{X})$ is finitely generated and torsion-free. Hence $\operatorname{Br}(X) / \operatorname{Br}_{0}(X)$ is finite. The sum of local invariants of a given element of $\operatorname{Br}(X)$ is a continuous function on $X\left(\mathbb{A}_{k}\right)$ with finitely many values, and this function is identically zero if the element is in $\operatorname{Br}_{0}(X)$. The lemma follows.

By the main result of [17] the conditions of this lemma are satisfied for $K 3$ surfaces. See Section 4 for a discussion of the finiteness of $\operatorname{Br}(X) / \operatorname{Br}_{1}(X)$.

Let us write $X_{v}=X \times_{k} k_{v}$ and, for $v \notin S, \mathcal{X}_{v}=\mathcal{X} \times \mathcal{O}_{S} \mathcal{O}_{v}$.

Lemma 1.2. We keep the assumptions of Lemma 1.1. If for every $v \notin S$ the image of $\operatorname{Br}(X) \rightarrow \operatorname{Br}\left(X_{v}\right)$ is contained in the subgroup generated by the images of $\operatorname{Br}\left(k_{v}\right)$ and $\operatorname{Br}\left(\mathcal{X}_{v}\right)$, then the answer to Question 1 is positive.

Proof. We may assume $X\left(\mathbb{A}_{k}\right)^{\mathrm{Br}} \neq \emptyset$. Fix $M=\left\{M_{v}\right\} \in X\left(\mathbb{A}_{k}\right)^{\mathrm{Br}}$. From the inclusion

$$
\operatorname{Br}(k) \hookrightarrow \bigoplus_{\text {all } v} \operatorname{Br}\left(k_{v}\right)
$$

provided by class field theory, and the fact that $X\left(\mathbb{A}_{k}\right)$ is non-empty we conclude that the natural map $\operatorname{Br}(k) \rightarrow \operatorname{Br}(X)$ is injective, so that $\operatorname{Br}(k)=\operatorname{Br}_{0}(X)$. Moreover, the exact sequence of abelian groups

$$
0 \rightarrow \operatorname{Br}(k) \rightarrow \operatorname{Br}(X) \rightarrow \operatorname{Br}(X) / \operatorname{Br}(k) \rightarrow 0
$$

has a splitting defined by $M$. Indeed, the injective map $\operatorname{Br}(k) \rightarrow \operatorname{Br}(X)$ has a retraction $r$ sending $A \in \operatorname{Br}(X)$ to the unique element $r(A) \in \operatorname{Br}(k)$ whose image in 
$\operatorname{Br}\left(k_{v}\right)$ is $A\left(M_{v}\right)$ for every $v$. Let us denote by $B$ the kernel of $r: \operatorname{Br}(X) \rightarrow \operatorname{Br}(k)$. An element $A \in \operatorname{Br}(X)$ lies in $B$ if and only if $A\left(M_{v}\right)=0$ for all $v$. Then $\operatorname{Br}(X)=\operatorname{Br}(k) \oplus B$, and $B$ projects isomorphically onto $\operatorname{Br}(X) / \operatorname{Br}(k)$.

To complete the proof it is enough to show that for any $v \notin S$, any $A \in B$ and any $N \in X\left(k_{v}\right)$ we have $A(N)=0 \in \operatorname{Br}\left(k_{v}\right)$. By assumption the image of $A$ in $\operatorname{Br}\left(X_{v}\right)$ can be written as a sum $A_{1}+A_{2}$, where $A_{1} \in \operatorname{Br}\left(\mathcal{X}_{v}\right)$ and $A_{2} \in \operatorname{Br}\left(k_{v}\right)$. Since $\mathcal{X}_{v}$ is proper over $\operatorname{Spec}\left(\mathcal{O}_{v}\right)$ we have $X\left(k_{v}\right)=\mathcal{X}_{v}\left(\mathcal{O}_{v}\right)$; hence $A_{1}(N) \in \operatorname{Br}\left(\mathcal{O}_{v}\right)=0$ for any $N \in X\left(k_{v}\right)$. We have $A\left(M_{v}\right)=0$ because $A \in B$. It follows that the 'constant' algebra $A_{2}$ has value 0 at $M_{v}$; hence $A_{2}=0$. We have proved that $A(N)=0 \in \operatorname{Br}\left(k_{v}\right)$, and the lemma follows.

The following well-known lemma is due to Grothendieck. It is given here for the ease of reference.

Lemma 1.3. Let $X$ be a smooth, projective and geometrically integral variety over a field of characteristic 0 . Assume that $\mathrm{H}^{1}\left(X, O_{X}\right)=\mathrm{H}^{2}\left(X, O_{X}\right)=0$ and that the Néron-Severi group $\mathrm{NS}(\bar{X})$ is torsion-free.

(i) Then the groups $\operatorname{Br}(\bar{X})$ and $\operatorname{Br}(X) / \operatorname{Br}_{0}(X)$ are finite.

(ii) We have $\operatorname{Br}(\bar{X})=0$ if and only if $\mathrm{H}_{e ̂ t}^{3}\left(\bar{X}, \mathbb{Z}_{\ell}(1)\right)_{\text {tors }}=0$ for every prime $\ell$. In this case $\operatorname{Br}_{1}(X)=\operatorname{Br}(X)$.

(iii) If $\operatorname{dim} X=2$, then $\operatorname{Br}(\bar{X})=0$ and $\operatorname{Br}_{1}(X)=\operatorname{Br}(X)$.

Proof. By [8, III.8] (more precisely, [8, III, (8.7), (8.9)]), we have a short exact sequence

$$
0 \longrightarrow(\mathbb{Q} / \mathbb{Z})^{b_{2}-\rho} \longrightarrow \operatorname{Br}(\bar{X}) \longrightarrow \bigoplus_{\ell} \mathrm{H}_{\text {ét }}^{3}\left(\bar{X}, \mathbb{Z}_{\ell}(1)\right)_{\text {tors }} \longrightarrow 0,
$$

where $b_{2}$ is the second Betti number of $\bar{X}$, and $\rho=\operatorname{rkNS}(\bar{X})$. Since the ground field $k$ has characteristic zero, for almost all $\ell$ the group $\mathrm{H}_{\text {ét }}^{3}\left(\bar{X}, \mathbb{Z}_{\ell}(1)\right)$ is torsionfree: this is a consequence of the comparison theorem between étale cohomology and Betti cohomology; see [13, Thm. III.3.12]. Thus the direct sum is finite. By Hodge theory the condition $\mathrm{H}^{2}\left(X, O_{X}\right)=0$ implies $\rho=b_{2}$. This shows that $\operatorname{Br}(\bar{X})$ is finite. As recalled above, the quotient $\operatorname{Br}_{1}(X) / \operatorname{Br}_{0}(X)$ is a subgroup of $\mathrm{H}^{1}(k, \operatorname{Pic}(\bar{X}))$. The hypothesis $\mathrm{H}^{1}\left(X, O_{X}\right)=0$ implies that the Picard variety of $X$ is trivial; hence $\operatorname{Pic}(\bar{X}) \simeq \operatorname{NS}(\bar{X})$ is finitely generated. In our case this group is torsion-free. It follows that $\mathrm{H}^{1}(k, \operatorname{Pic}(\bar{X}))$ is finite; thus $\operatorname{Br}(X) / \operatorname{Br}_{0}(X)$ is also finite. This proves (i) and (ii).

When $\operatorname{dim} X=2$, for every prime $\ell$ there is a perfect duality pairing of finite abelian groups

$$
\mathrm{H}_{\text {ét }}^{3}\left(\bar{X}, \mathbb{Z}_{\ell}(1)\right)\{\ell\} \times \mathrm{NS}(\bar{X})\{\ell\} \longrightarrow \mathbb{Q}_{\ell} / \mathbb{Z}_{\ell}
$$

see [8, III, (8.10), (8.11)]. Thus (iii) follows from (ii).

\section{Brauer group of A VARIETy OVER A LOCAL FIELD AND BRAUER PAIRING}

Throughout this section we use the following notation:

$K$ is a finite extension of $\mathbb{Q}_{p}$,

$\bar{K}$ is an algebraic closure of $K$,

$K_{\mathrm{nr}} \subset \bar{K}$ is the maximal unramified extension,

$\mathfrak{g}=\operatorname{Gal}(\bar{K} / K), \quad G=\operatorname{Gal}\left(K_{\mathrm{nr}} / K\right), \quad I=\operatorname{Gal}\left(\bar{K} / K_{\mathrm{nr}}\right)$,

$R$ is the ring of integers of $K$,

$R_{\mathrm{nr}}$ is the ring of integers of $K_{\mathrm{nr}}$, 
$X$ is a proper, smooth and geometrically integral variety over $K$, $\pi: \mathcal{X} \rightarrow \operatorname{Spec}(R)$ is a faithfully flat proper morphism with $X=\mathcal{X} \times{ }_{R} K$, $X_{\mathrm{nr}}=X \times_{K} K_{\mathrm{nr}}, \bar{X}=X \times_{K} \bar{K}, \mathcal{X}_{\mathrm{nr}}=\mathcal{X} \times_{R} R_{\mathrm{nr}}$.

We shall constantly use the classical result that $\operatorname{Br}\left(K_{\mathrm{nr}}\right)=0$, which implies that $\mathrm{H}^{2}\left(G, K_{\mathrm{nr}}^{*}\right)=\operatorname{Br}(K)$; see [14, Ch. XII, Thm. 1 and its corollary.

The following finiteness statement holds without a good reduction hypothesis. It will not be used in this note.

Proposition 2.1. For any smooth, proper and geometrically integral variety $X$ over $K$, the group $\operatorname{Ker}\left[\operatorname{Br}(X) \rightarrow \operatorname{Br}\left(X_{\mathrm{nr}}\right)\right] / \operatorname{Br}_{0}(X)$ is finite.

Proof. There is a Hochschild-Serre spectral sequence attached to the morphism $X_{\mathrm{nr}} \rightarrow X:$

$$
E_{2}^{p q}=\mathrm{H}^{p}\left(G, \mathrm{H}_{\text {ét }}^{q}\left(X_{\mathrm{nr}}, \mathbb{G}_{m}\right)\right) \Rightarrow \mathrm{H}_{\text {ét }}^{p+q}\left(X, \mathbb{G}_{m}\right) .
$$

Since $\mathrm{H}^{2}\left(G, K_{\mathrm{nr}}^{*}\right)=\operatorname{Br}(K)$, the exact sequence of low-degree terms of (2) shows that the group under consideration is a subgroup of $\mathrm{H}^{1}\left(G, \operatorname{Pic}\left(X_{\mathrm{nr}}\right)\right)$. We have an exact sequence of continuous discrete $\mathfrak{g}$-modules

$$
0 \rightarrow \operatorname{Pic}^{0}(\bar{X}) \rightarrow \operatorname{Pic}(\bar{X}) \rightarrow \mathrm{NS}(\bar{X}) \rightarrow 0 .
$$

By the representability of the Picard functor over a field of characteristic zero (due to Weil and Grothendieck; see [5], Thm. 9.5.4 and Cor. 9.5.14) there exists an abelian variety $A$ over $K$ such that $A(\bar{K})$ is isomorphic to $\operatorname{Pic}^{0}(\bar{X})$ as a $\mathfrak{g}$-module. Thus we rewrite the previous sequence as

$$
0 \rightarrow A(\bar{K}) \rightarrow \operatorname{Pic}(\bar{X}) \rightarrow \mathrm{NS}(\bar{X}) \rightarrow 0 .
$$

The Hochschild-Serre spectral sequence attached to $\bar{X} \rightarrow X_{\mathrm{nr}}$ is

$$
E_{2}^{p q}=\mathrm{H}^{p}\left(I, \mathrm{H}_{\text {ét }}^{q}\left(\bar{X}, \mathbb{G}_{m}\right)\right) \Rightarrow \mathrm{H}_{\text {ét }}^{p+q}\left(X_{\mathrm{nr}}, \mathbb{G}_{m}\right) .
$$

By Hilbert's theorem 90 we have $\mathrm{H}^{1}\left(I, \bar{K}^{*}\right)=0$. Since $\operatorname{Br}\left(K_{\mathrm{nr}}\right)=0$ we obtain that the natural map $\operatorname{Pic}\left(X_{\mathrm{nr}}\right) \rightarrow \operatorname{Pic}(\bar{X})^{I}$ is an isomorphism. Now, by taking $I$-invariants in (3), we obtain the exact sequence of $G$-modules

$$
0 \rightarrow A\left(K_{\mathrm{nr}}\right) \rightarrow \operatorname{Pic}\left(X_{\mathrm{nr}}\right) \rightarrow \mathrm{NS}(\bar{X})^{I} .
$$

The group $\operatorname{NS}(\bar{X})$ is finitely generated by the theorem of Néron and Severi, hence so is $\operatorname{NS}(\bar{X})^{I}$. Thus there is a $G$-module $N$, finitely generated as an abelian group, that fits into the exact sequence of continuous discrete $G$-modules

$$
0 \rightarrow A\left(K_{\mathrm{nr}}\right) \rightarrow \operatorname{Pic}\left(X_{\mathrm{nr}}\right) \rightarrow N \rightarrow 0 .
$$

The resulting exact sequence of cohomology groups gives an exact sequence

$$
\mathrm{H}^{1}\left(G, A\left(K_{\mathrm{nr}}\right)\right) \rightarrow \mathrm{H}^{1}\left(G, \operatorname{Pic}\left(X_{\mathrm{nr}}\right)\right) \rightarrow \mathrm{H}^{1}(G, N) .
$$

We note that $G$ is canonically isomorphic to the profinite completion $\hat{\mathbb{Z}}$, with the Frobenius as a topological generator. If $M$ is a continuous discrete $G$-module which is finitely generated as an abelian group, then $\mathrm{H}^{1}(G, M)$ is finite. To see this, let $G^{\prime}$ be a finite index subgroup of $G$ that acts trivially on $M$. The group $G^{\prime} \simeq \hat{\mathbb{Z}}$ has a dense subgroup $\mathbb{Z}$ generated by a power of the Frobenius. Now $\mathrm{H}^{1}\left(G^{\prime}, M\right)$ is the group of continuous homomorphisms

$$
\operatorname{Hom}_{\text {cont }}\left(G^{\prime}, M\right)=\operatorname{Hom}_{\text {cont }}\left(G^{\prime}, M_{\text {tors }}\right)=M_{\text {tors }},
$$


which is visibly finite. An application of the restriction-inflation sequence finishes the proof of the finiteness of $\mathrm{H}^{1}(G, M)$.

To complete the proof of the proposition it remains to prove the finiteness of $\mathrm{H}^{1}\left(G, A\left(K_{\mathrm{nr}}\right)\right)$. By Prop. I.3.8 of [12] this group is isomorphic to $\mathrm{H}^{1}\left(G, \pi_{0}\left(A_{0}\right)\right)$, where $\pi_{0}\left(A_{0}\right)$ is the group of connected components of the closed fibre $A_{0}$ of the Néron model of $A$ over $\operatorname{Spec}(R)$. Since $\pi_{0}\left(A_{0}\right)$ is finite, we see that $\mathrm{H}^{1}\left(G, \pi_{0}\left(A_{0}\right)\right)$ is finite.

Particular cases of the following two results have been known for some time; see [1] and [2, Thm. 1].

Lemma 2.2. If $\mathcal{X}$ is smooth over $R$ with geometrically integral fibres, then

(i) the following natural map is surjective:

$$
\operatorname{Br}(K) \oplus \operatorname{Ker}\left[\operatorname{Br}(\mathcal{X}) \rightarrow \operatorname{Br}\left(\mathcal{X}_{\mathrm{nr}}\right)\right] \longrightarrow \operatorname{Ker}\left[\operatorname{Br}(X) \rightarrow \operatorname{Br}\left(X_{\mathrm{nr}}\right)\right] ;
$$

(ii) for any $A \in \operatorname{Ker}\left[\operatorname{Br}(X) \rightarrow \operatorname{Br}\left(X_{\mathrm{nr}}\right)\right]$ the image of the evaluation map $X(K) \rightarrow$ $\operatorname{Br}(K)$ given by $M \mapsto A(M)$ consists of one element.

Proof. The map in (i) is well defined since $\operatorname{Br}\left(K_{\mathrm{nr}}\right)=0$, so that the composition $\operatorname{Br}(K) \rightarrow \operatorname{Br}(X) \rightarrow \operatorname{Br}\left(X_{\text {nr }}\right)$ is zero.

The restriction map $\operatorname{Pic}\left(\mathcal{X}_{\mathrm{nr}}\right) \rightarrow \operatorname{Pic}\left(X_{\mathrm{nr}}\right)$ is surjective since $\mathcal{X}_{\mathrm{nr}}$ is regular. The kernel of this map is generated by the classes of components of the closed fibre of $\mathcal{X}_{\mathrm{nr}} \rightarrow \operatorname{Spec}(R)$. The closed fibre is a principal divisor in $\mathcal{X}_{\mathrm{nr}}$; since it is integral, the restriction map gives an isomorphism of $G$-modules

$$
\operatorname{Pic}\left(\mathcal{X}_{\mathrm{nr}}\right) \stackrel{\sim}{\longrightarrow} \operatorname{Pic}\left(X_{\mathrm{nr}}\right) \text {. }
$$

There is a Hochschild-Serre spectral sequence attached to the morphism $\mathcal{X}_{\mathrm{nr}} \rightarrow \mathcal{X}$ :

$$
E_{2}^{p q}=\mathrm{H}^{p}\left(G, \mathrm{H}_{\text {ét }}^{q}\left(\mathcal{X}_{\mathrm{nr}}, \mathbb{G}_{m}\right)\right) \Rightarrow \mathrm{H}_{\text {ét }}^{p+q}\left(\mathcal{X}, \mathbb{G}_{m}\right),
$$

and a similar sequence (2) attached to the morphism $X_{\mathrm{nr}} \rightarrow X$. By functoriality the maps in (6) and (2) are compatible with the inclusion of the generic fibres $X \hookrightarrow \mathcal{X}$ and $X_{\mathrm{nr}} \hookrightarrow \mathcal{X}_{\mathrm{nr}}$. We have $\mathrm{H}_{\text {ét }}^{0}\left(\mathcal{X}_{\mathrm{nr}}, \mathbb{G}_{m}\right)=R_{\mathrm{nr}}^{*}$ because $\pi: \mathcal{X} \rightarrow \operatorname{Spec}(R)$ is proper with geometrically integral fibres. The low-degree terms of the two spectral sequences give rise to the following commutative diagram of exact sequences, where the equality is induced by (5):

$$
\begin{array}{rlrrrrr}
\mathrm{H}^{2}\left(G, R_{\mathrm{nr}}^{*}\right) & \rightarrow & \operatorname{Ker}\left[\operatorname{Br}(\mathcal{X}) \rightarrow \operatorname{Br}\left(\mathcal{X}_{\mathrm{nr}}\right)\right] & \rightarrow & \mathrm{H}^{1}\left(G, \operatorname{Pic}\left(\mathcal{X}_{\mathrm{nr}}\right)\right) & \rightarrow & \mathrm{H}^{3}\left(G, R_{\mathrm{nr}}^{*}\right) \\
\downarrow & & \downarrow & \| & & & \downarrow \\
\mathrm{H}^{2}\left(G, K_{\mathrm{nr}}^{*}\right) & \rightarrow & \operatorname{Ker}\left[\operatorname{Br}(X) \rightarrow \operatorname{Br}\left(X_{\mathrm{nr}}\right)\right] & \rightarrow & \mathrm{H}^{1}\left(G, \operatorname{Pic}\left(X_{\mathrm{nr}}\right)\right) & \rightarrow & \mathrm{H}^{3}\left(G, K_{\mathrm{nr}}^{*}\right) .
\end{array}
$$

We have $\mathrm{H}^{3}\left(G, R_{\mathrm{nr}}^{*}\right)=0$, since the group $G \cong \hat{\mathbb{Z}}$ has strict cohomological dimension 2 . Since $\mathrm{H}^{2}\left(G, K_{\mathrm{nr}}^{*}\right)=\operatorname{Br}(K)$, the statement of (i) follows from the above diagram.

Any element $A \in \operatorname{Br}(\mathcal{X}) \subset \operatorname{Br}(X)$ vanishes on $\mathcal{X}(R)=X(K)$, since it takes values in $\operatorname{Br}(R)=0$. This proves (ii).

Proposition 2.3. Assume that $\mathcal{X}$ is smooth over $R$ with geometrically integral fibres, $\mathrm{H}^{1}\left(X, O_{X}\right)=0$ and the Néron-Severi group $\mathrm{NS}(\bar{X})$ is torsion-free.

(i) Then the quotient $\operatorname{Br}_{1}(X) / \operatorname{Br}_{0}(X)$ is finite, and every element of $\operatorname{Br}_{1}(X) \subset$ $\operatorname{Br}(X)$ can be written as $\alpha+\beta$, where $\alpha \in \operatorname{Br}_{0}(X)$ and $\beta \in \operatorname{Br}(\mathcal{X}) \subset \operatorname{Br}(X)$. In particular, for any $A \in \mathrm{Br}_{1}(X)$ the image of the evaluation map $X(K) \rightarrow \operatorname{Br}(K)$ given by $M \mapsto A(M)$ consists of one element. 
(ii) Assume, moreover, that $\mathrm{H}^{2}\left(X, O_{X}\right)=0$, and that for every prime $\ell$ the group $\mathrm{H}_{e ́ t}^{3}\left(\bar{X}, \mathbb{Z}_{\ell}\right)$ is torsion-free. Then the quotient $\operatorname{Br}(X) / \operatorname{Br}_{0}(X)$ is finite and generated by the image of $\operatorname{Br}(\mathcal{X})$.

Proof. For $\ell \neq p$ the smooth base change theorem in étale cohomology for the smooth and proper morphism $\pi: \mathcal{X} \rightarrow \operatorname{Spec}(R)$ implies that the natural action of the inertia subgroup $I$ on $\mathrm{H}_{\text {ét }}^{2}\left(\bar{X}, \mathbb{Z}_{\ell}(1)\right)$ is trivial. Indeed, by [13, Cor. VI.4.2] the étale sheaf $R^{2} \pi_{*} \mu_{\ell^{m}}$ is locally constant for every $m \geq 1$. Also, the fibre of $R^{2} \pi_{*} \mu_{\ell^{m}}$ at the generic geometric point $\operatorname{Spec}(\bar{K}) \rightarrow \operatorname{Spec}(R)$ is $\mathrm{H}_{\text {ét }}^{2}\left(\bar{X}, \mu_{\ell^{m}}\right)$. Now it follows from Remark 1.2 (b) in [13, Ch. V] that the action of $\mathfrak{g}$ on $\mathrm{H}_{\text {ét }}^{2}\left(\bar{X}, \mu_{\ell^{m}}\right)$ factors through

$$
\pi_{1}(\operatorname{Spec}(R), \operatorname{Spec}(\bar{K}))=\operatorname{Gal}\left(K_{\mathrm{nr}} / K\right)=G=\mathfrak{g} / I ;
$$

see [13, Ex. I.5.2(b)]. Thus $I$ acts trivially on $\mathrm{H}_{\text {ét }}^{2}\left(\bar{X}, \mu_{\ell^{m}}\right)$ for every $m$; hence $I$ acts trivially on $\mathrm{H}_{\text {ét }}^{2}\left(\bar{X}, \mathbb{Z}_{\ell}(1)\right)$.

Since $K$ has characteristic zero, for any prime $\ell$ the Kummer sequence gives a Galois equivariant embedding

$$
\mathrm{NS}(\bar{X}) \otimes \mathbb{Z}_{\ell} \hookrightarrow \mathrm{H}_{\text {ét }}^{2}\left(\bar{X}, \mathbb{Z}_{\ell}(1)\right)
$$

For any $\ell \neq p$ we conclude that $I$ acts trivially on $\mathrm{NS}(\bar{X}) \otimes \mathbb{Z}_{\ell}$. Hence $I$ acts trivially on the quotient of the finitely generated group $\mathrm{NS}(\bar{X})$ by its $p$-torsion subgroup.

So far the arguments apply to any smooth proper $R$-scheme with geometrically integral fibres.

If $\mathrm{NS}(\bar{X})$ is torsion-free, then we have $\mathrm{NS}(\bar{X}) \subset \mathrm{NS}(\bar{X}) \otimes \mathbb{Z}_{\ell}$; thus the natural action of $I$ on $\mathrm{NS}(\bar{X})$ is also trivial. Under the hypothesis $\mathrm{H}^{1}\left(X, O_{X}\right)=0$, we now conclude that $\operatorname{Pic}(\bar{X}) \simeq \operatorname{NS}(\bar{X})$ is a finitely generated torsion-free abelian group with trivial action of inertia. This implies

$$
\mathrm{H}^{1}(I, \operatorname{Pic}(\bar{X}))=0 .
$$

It also implies that $\mathrm{H}^{1}(k, \operatorname{Pic}(\bar{X}))$ is finite. Thus $\operatorname{Br}_{1}(X) / \operatorname{Br}_{0}(X)$ is finite.

We have the following commutative diagram of exact sequences:

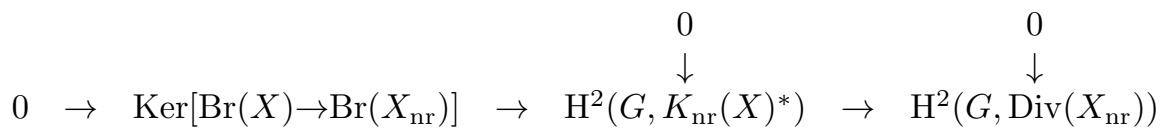

$$
\begin{aligned}
& 0 \rightarrow \operatorname{Ker}[\operatorname{Br}(X) \rightarrow \operatorname{Br}(\bar{X})] \quad \rightarrow \quad \mathrm{H}^{2}\left(\mathfrak{g}, \stackrel{\downarrow}{K}(X)^{*}\right) \quad \rightarrow \quad \mathrm{H}^{2}(\mathfrak{g}, \stackrel{\downarrow}{\operatorname{Div}(\bar{X}))} \\
& \mathrm{H}^{2}\left(I, \frac{\downarrow}{K}(X)^{*}\right) \rightarrow \mathrm{H}^{2}(I, \stackrel{\operatorname{Div}}{\downarrow}(\bar{X})) \text {. }
\end{aligned}
$$

The top and middle horizontal sequences are special cases of an exact sequence associated to a smooth variety over a field $K$ and a Galois extension of $K$; see [3, §1.5.0]. The two vertical sequences are restriction-inflation sequences. Their exactness follows from $\mathrm{H}^{1}\left(I, \bar{K}(X)^{*}\right)=0$ (Hilbert's theorem 90) and from $\mathrm{H}^{1}(I, \operatorname{Div}(\bar{X}))=0$ (since $X$ is smooth, the $I$-module $\operatorname{Div}(\bar{X})$ is a permutation module; the vanishing then follows from Shapiro's lemma).

As mentioned above, under our hypotheses, $\mathrm{H}^{1}(I, \operatorname{Pic}(\bar{X}))=0$. We also have $\mathrm{H}^{2}\left(I, \bar{K}^{*}\right)=\operatorname{Br}\left(K_{\mathrm{nr}}\right)=0$. The exact sequence

$$
0 \rightarrow \bar{K}^{*} \rightarrow \bar{K}(X)^{*} \rightarrow \operatorname{Div}(\bar{X}) \rightarrow \operatorname{Pic}(\bar{X}) \rightarrow 0
$$


then shows that the natural map $\mathrm{H}^{2}\left(I, \bar{K}(X)^{*}\right) \rightarrow \mathrm{H}^{2}(I, \operatorname{Div}(\bar{X}))$ is injective. From the commutative diagram above we conclude that the following natural inclusion is an isomorphism:

$$
\operatorname{Ker}\left[\operatorname{Br}(X) \rightarrow \operatorname{Br}\left(X_{\mathrm{nr}}\right)\right] \stackrel{\sim}{\longrightarrow} \operatorname{Ker}[\operatorname{Br}(X) \rightarrow \operatorname{Br}(\bar{X})] .
$$

An application of Lemma 2.2 concludes the proof of statement (i).

Statement (ii) follows from (i) and Lemma 1.3 (ii).

We now would like to explore the situation when $\mathrm{H}^{2}\left(X, O_{X}\right)$ is not necessarily zero, so we must take into account the transcendental Brauer group as well.

Proposition 2.4. Let $\ell$ be a prime, $\ell \neq p$. Assume that $\mathcal{X}$ is smooth over $R$ with geometrically integral fibres, and that the closed geometric fibre has no connected unramified cyclic covering of degree $\ell$.

(i) Then the group $\operatorname{Br}(X)\{\ell\}$ is generated by the images of $\operatorname{Br}(\mathcal{X})\{\ell\}$ and $\operatorname{Br}(K)\{\ell\}$.

(ii) If $X(K) \neq \emptyset$, then for any $A \in \operatorname{Br}(X)\{\ell\}$ the image of the evaluation map $X(K) \rightarrow \operatorname{Br}(K)$ given by $M \mapsto A(M)$ consists of one element.

Proof. (i) Let $\mathbb{F}$ be the residue field of $R$, and let $\mathcal{X}_{0}=\mathcal{X} \times{ }_{R} \mathbb{F}$ be the closed fibre of $\pi$. By a special case of a result of K. Kato [10, Prop. 1.7], to whose paper we refer for the explicit description of the maps involved, for any positive integer $n$ there is a natural complex

$$
\operatorname{Br}(X)\left[\ell^{n}\right] \stackrel{\text { res }}{\longrightarrow} \mathrm{H}^{1}\left(\mathbb{F}\left(\mathcal{X}_{0}\right), \mathbb{Z} / \ell^{n}\right) \longrightarrow \bigoplus_{Y \subset \mathcal{X}_{0}} \mathrm{H}^{0}\left(\mathbb{F}(Y), \mathbb{Z} / \ell^{n}(-1)\right),
$$

where $Y$ ranges over closed integral subvarieties of codimension 1 in $\mathcal{X}_{0}$, the field $\mathbb{F}\left(\mathcal{X}_{0}\right)$ is the function field of $\mathcal{X}_{0}$, and $\mathbb{F}(Y)$ is the function field of $Y$. The elements of $\mathrm{H}^{1}\left(\mathbb{F}\left(\mathcal{X}_{0}\right), \mathbb{Z} / \ell^{n}\right)$ correspond to characters of the absolute Galois group of $\mathbb{F}\left(\mathcal{X}_{0}\right)$ with values in $\mathbb{Z} / \ell^{n}$. These correspond to connected cyclic coverings $W \rightarrow \mathcal{X}_{0}$ (not necessary unramified) of degree dividing $\ell^{n}$, where $W$ is an irreducible normal variety. From Kato's complex it follows that for any $A \in \operatorname{Br}(X)\left[\ell^{n}\right]$ the residue $\operatorname{res}(A) \in \mathrm{H}^{1}\left(\mathbb{F}\left(\mathcal{X}_{0}\right), \mathbb{Z} / \ell^{n}\right)$ is unramified in codimension 1. Thus the corresponding covering $W \rightarrow \mathcal{X}_{0}$ is a covering of a smooth scheme unramified at all the points of codimension 1 , hence is an étale covering of $\mathcal{X}_{0}$ by the Zariski-Nagata purity theorem (SGA $1 \mathrm{X}$, Théorème 3.1). In other words,

$$
\operatorname{res}(A) \in \mathrm{H}_{\text {ét }}^{1}\left(\mathcal{X}_{0}, \mathbb{Z} / \ell^{n}\right) \subset \mathrm{H}^{1}\left(\mathbb{F}\left(\mathcal{X}_{0}\right), \mathbb{Z} / \ell^{n}\right) .
$$

Let $\overline{\mathcal{X}}_{0}=\mathcal{X}_{0} \times_{\mathbb{F}} \overline{\mathbb{F}}$, where $\overline{\mathbb{F}}$ is an algebraic closure of $\mathbb{F}$. The spectral sequence

$$
E_{2}^{p q}=\mathrm{H}^{p}\left(\mathbb{F}, \mathrm{H}_{\text {ét }}^{q}\left(\overline{\mathcal{X}}_{0}, \mathbb{Z} / \ell^{n}\right)\right) \Rightarrow \mathrm{H}_{\text {ét }}^{p+q}\left(\mathcal{X}_{0}, \mathbb{Z} / \ell^{n}\right)
$$

gives rise to the exact sequence

$$
0 \rightarrow \mathrm{H}^{1}\left(\mathbb{F}, \mathbb{Z} / \ell^{n}\right) \rightarrow \mathrm{H}_{\text {ét }}^{1}\left(\mathcal{X}_{0}, \mathbb{Z} / \ell^{n}\right) \rightarrow \mathrm{H}_{\text {ét }}^{1}\left(\overline{\mathcal{X}}_{0}, \mathbb{Z} / \ell^{n}\right) .
$$

Since $\overline{\mathcal{X}}_{0}$ has no connected unramified cyclic covering of degree $\ell$, we have $\mathrm{H}_{\text {ét }}^{1}\left(\overline{\mathcal{X}}_{0}, \mathbb{Z} / \ell^{n}\right)=0$. Thus $\operatorname{res}(A)$ belongs to the injective image of $\mathrm{H}^{1}\left(\mathbb{F}, \mathbb{Z} / \ell^{n}\right)$ in $\mathrm{H}^{1}\left(\mathcal{X}_{0}, \mathbb{Z} / \ell^{n}\right)$.

By local class field theory the residue map $\operatorname{Br}(K)\left[\ell^{n}\right] \rightarrow \mathrm{H}^{1}\left(\mathbb{F}, \mathbb{Z} / \ell^{n}\right)$ is an isomorphism. Hence for any $A \in \operatorname{Br}(X)\{\ell\}$ there exists $\alpha \in \operatorname{Br}(K)\{\ell\}$ such that the residue of $A-\alpha$ at any point of codimension 1 of $\mathcal{X}$ is zero. By Gabber's absolute 
purity theorem [6], this implies that $A-\alpha$ belongs to $\operatorname{Br}(\mathcal{X})\{\ell\} \subset \operatorname{Br}(X)\{\ell\}$. This completes the proof of (i).

Since $\mathcal{X}$ is proper over $\operatorname{Spec}(R)$ we have $X(K)=\mathcal{X}(R)$. Statement (ii) follows because $\operatorname{Br}(R)=0$.

Remarks. 1. Already for $\pi$ smooth and proper, it is an interesting $p$-adic problem to decide whether there is an analogous proposition for $\operatorname{Br}(X)\{p\}$. For algebras split by an unramified extension of $K$, including those of order divisible by $p$, this follows from Lemma 2.2 (see also [1, Prop. 6]).

2. When $\mathcal{X}$ has dimension at most 3, we may refer to Gabber's earlier purity theorem [7, Thm. $\left.2^{\prime}\right]$ rather than to [6.

3. The hypotheses of Proposition 2.4 apply in particular when the fibres of $\pi$ : $\mathcal{X} \rightarrow \operatorname{Spec}(R)$ are smooth complete intersections of dimension at least 2 in projective space (an application of the weak Lefschetz theorem in étale cohomology; see [11]). In particular they apply to smooth surfaces of arbitrary degree in $\mathbb{P}^{3}$.

Remark (A remark on the bad reduction case). Let $\ell$ be a prime, $\ell \neq p$. Assume that $\mathcal{X}$ is a regular scheme, and $X(K)=\mathcal{X}(R) \neq \emptyset$. Let $\mathcal{Z}$ be the largest open subscheme of $\mathcal{X}$ smooth over $\operatorname{Spec}(R)$, such that $\mathcal{Z} \times{ }_{R} K=X$, and every irreducible component of the closed fibre $\mathcal{Z}_{0}$ is geometrically irreducible. Since $\mathcal{X}$ is regular, a well-known valuation argument shows that $X(K)=\mathcal{X}(R)=\mathcal{Z}(R)$; see, e.g., [15, the proof of Lemma 1.1 (b). Let $V_{1}, \ldots, V_{n}$ be the irreducible components of $\mathcal{Z}_{0}$, and let $\bar{V}_{i}=V_{i} \times_{\mathbb{F}} \overline{\mathbb{F}}$. Then Kato's complex for $\mathcal{Z}$ has the form

$$
\operatorname{Br}(X)\left[\ell^{n}\right] \stackrel{\text { res }}{\longrightarrow} \bigoplus_{i=1}^{n} \mathrm{H}^{1}\left(\mathbb{F}\left(V_{i}\right), \mathbb{Z} / \ell^{n}\right) \longrightarrow \bigoplus_{Y \subset \mathcal{Z}_{0}} \mathrm{H}^{0}\left(\mathbb{F}(Y), \mathbb{Z} / \ell^{n}(-1)\right) .
$$

We see that for any $A \in \operatorname{Br}(X)\left[\ell^{n}\right]$ the residue $\operatorname{res}_{i}(A) \in \mathrm{H}^{1}\left(\mathbb{F}\left(V_{i}\right), \mathbb{Z} / \ell^{n}\right)$ belongs to the subgroup $\mathrm{H}^{1}\left(V_{i}, \mathbb{Z} / \ell^{n}\right)$. We note that this group is finite. This follows from the exact sequence

$$
0 \rightarrow \mathrm{H}^{1}\left(\mathbb{F}, \mathrm{H}^{0}\left(\bar{V}_{i}, \mathbb{Z} / \ell^{n}\right)\right) \rightarrow \mathrm{H}^{1}\left(V_{i}, \mathbb{Z} / \ell^{n}\right) \rightarrow \mathrm{H}^{1}\left(\bar{V}_{i}, \mathbb{Z} / \ell^{n}\right),
$$

and the fact that $\mathrm{H}^{1}\left(\hat{\mathbb{Z}}, \mathbb{Z} / \ell^{n}\right) \simeq \mathbb{Z} / \ell^{n}$. We conclude that $\operatorname{res}_{i}$, as a function on $\operatorname{Br}(X)\left[\ell^{n}\right]$, takes only finitely many values.

If the reduction $\tilde{M}$ of $M \in X(R)$ belongs to $V_{i}$, then the local invariant of $A(M) \in \operatorname{Br}(K)$ is the pullback of $\operatorname{res}_{i}(A)$ under the natural map $\mathrm{H}^{1}\left(V_{i}, \mathbb{Z} / \ell^{n}\right) \rightarrow$ $\mathrm{H}^{1}\left(\mathbb{F}, \mathbb{Z} / \ell^{n}\right)$ defined by $\tilde{M}$. Combining this information for all irreducible components of $\mathcal{Z}_{0}$ we obtain a finite partition of the set $X(K)$ such that for any $A \in \operatorname{Br}(X)\left[\ell^{n}\right]$ the image of the evaluation map $X(K) \rightarrow \operatorname{Br}(K)$ given by $M \mapsto A(M)$ is constant on each element of the partition.

\section{Structure of the Brauer-Manin Set over a Number field}

We are now ready to prove the main results of this note.

Theorem 3.1. Let $k$ be a number field. Let $S$ be a finite set of places of $k$ containing the archimedean places, and let $\mathcal{O}_{S}$ be the subring of $k$ consisting of the elements that are integral at the primes not in $S$. Let $\pi: \mathcal{X} \rightarrow \operatorname{Spec}\left(\mathcal{O}_{S}\right)$ be a smooth proper $\mathcal{O}_{S}$-scheme with geometrically integral fibres. Let $X / k$ be its generic fibre. Assume

(i) $\mathrm{H}^{1}\left(X, O_{X}\right)=0$;

(ii) the Néron-Severi group $\mathrm{NS}(\bar{X})$ has no torsion;

(iii) $\operatorname{Br}(X) / \operatorname{Br}_{1}(X)$ is a finite abelian group of order invertible in $\mathcal{O}_{S}$. Then the answer to Question 1 in the Introduction is in the affirmative. 
Proof. Let $v$ be a place of $k$ not contained in $S$, and let $p$ be the residual characteristic of $k_{v}$. Assumption (iii) implies that $\operatorname{Br}(X)\{p\} \subset \operatorname{Br}_{1}(X)$. Then, by Proposition 2.3, the image of $\operatorname{Br}(X)\{p\}$ in $\operatorname{Br}\left(X_{v}\right)$ is contained in the subgroup generated by the images of $\operatorname{Br}\left(k_{v}\right)$ and $\operatorname{Br}\left(\mathcal{X}_{v}\right)$. By Proposition 2.4 the same is true for $\operatorname{Br}(X)\{\ell\}$ for any prime $\ell$ not equal to $p$. For this we only need to check that $\mathrm{H}_{\text {ét }}^{1}\left(\overline{\mathcal{X}}_{M}, \mathbb{Z} / \ell\right)=0$, where $\overline{\mathcal{X}}_{M}$ is the closed geometric fibre of $\pi: \mathcal{X}_{v} \rightarrow \operatorname{Spec}\left(\mathcal{O}_{v}\right)$. By the smooth base changetheorem for étale cohomology (see, e.g. 13, VI, Cor. 4.2) the group $\mathrm{H}_{\text {êt }}^{1}\left(\overline{\mathcal{X}}_{M}, \mathbb{Z} / \ell\right)$ is isomorphic to $\mathrm{H}_{\text {ét }}^{1}\left(\bar{X}_{v}, \mathbb{Z} / \ell\right)$, which in turn is isomorphic to $\mathrm{H}_{\text {ét }}^{1}(\bar{X}, \mathbb{Z} / \ell)$ by [13], VI, Cor. 4.3. The Kummer exact sequence gives an isomorphism $\mathrm{H}_{\text {ét }}^{1}\left(\bar{X}, \mu_{\ell}\right) \stackrel{\sim}{\longrightarrow} \operatorname{Pic}(\bar{X})[\ell]$, and the vanishing of the latter group follows from conditions (i) and (ii). The statement now follows from Lemma 1.2

Corollary 3.2. Let $\pi: \mathcal{X} \rightarrow \operatorname{Spec}\left(\mathcal{O}_{S}\right)$ be a smooth proper $\mathcal{O}_{S}$-scheme with geometrically integral fibres. Let $X / k$ be its generic fibre. Assume

(i) $\mathrm{H}^{i}\left(X, O_{X}\right)=0$ for $i=1,2$;

(ii) the Néron-Severi group $\mathrm{NS}(\bar{X})$ has no torsion;

(iii) either $\operatorname{dim} X=2$, or $\mathrm{H}_{e t}^{3}\left(\bar{X}, \mathbb{Z}_{\ell}\right)$ is torsion-free for every prime $\ell$ outside $S$. Then the answer to Question 1 is in the affirmative.

Proof. This follows from Theorem 3.1 by Lemma 1.3 and its proof.

This corollary can be applied to rationally connected varieties. Indeed, over a field of characteristic zero, these varieties are $O_{X}$-acyclic and algebraically simply connected [4, Cor. 4.18].

When we no longer have $\mathrm{H}^{2}\left(X, O_{X}\right)=0$, condition (iii) in Theorem 3.1 is not easy to check in general. However, this can be done in an important particular case: as an application of rather delicate computations of 9 we now show that the answer to Question 1 is positive for smooth diagonal quartics over $\mathbb{Q}$, so that only the real place and the primes of bad reduction can show up in the Brauer-Manin obstruction.

Corollary 3.3. Let $D$ be the diagonal quartic surface over $\mathbb{Q}$ given by

$$
x_{0}^{4}+a_{1} x_{1}^{4}+a_{2} x_{2}^{4}+a_{3} x_{3}^{4}=0,
$$

where $a_{1}, a_{2}, a_{3} \in \mathbb{Q}^{*}$. Let $\mathcal{S}$ be the set of primes consisting of 2 and the primes dividing the numerators or the denominators of $a_{1}, a_{2}, a_{3}$. Let $Z$ be the image of the projection

$$
D\left(\mathbb{A}_{\mathbb{Q}}\right)^{\mathrm{Br}} \rightarrow D(\mathbb{R}) \times \prod_{p \in \mathcal{S}} D\left(\mathbb{Q}_{p}\right) .
$$

Then $D\left(\mathbb{A}_{\mathbb{Q}}\right)^{\mathrm{Br}}=Z \times \prod_{p \notin \mathcal{S}} D\left(\mathbb{Q}_{p}\right)$.

Proof. $D$ is a $K 3$ surface, for which the geometric conditions (i) and (ii) of Theorem 3.1 are well known. By Theorem 3.2 of [9], only the primes from $\{2,3,5\} \cap \mathcal{S}$ can divide the order of the finite group $\operatorname{Br}(D) / \mathrm{Br}_{1}(D)$.

There are other $K 3$ surfaces over $\mathbb{Q}$ to which Theorem 3.1 can be applied. Let $X$ be the Kummer surface attached to the product of elliptic curves $E$ and $E^{\prime}$ over $\mathbb{Q}$. By [18, Prop. 1.4] we have

$$
\operatorname{Br}(\bar{X})^{\Gamma} \cong \operatorname{Br}\left(\bar{E} \times \bar{E}^{\prime}\right)^{\Gamma},
$$


where $\Gamma=\operatorname{Gal}(\overline{\mathbb{Q}} / \mathbb{Q})$. If $E$ and $E^{\prime}$ are not isogenous over $\overline{\mathbb{Q}}$, then by [18, Prop. 3.1] for any integer $n$ we have

$$
\operatorname{Br}\left(\bar{E} \times \bar{E}^{\prime}\right)[n]^{\Gamma} \cong \operatorname{Hom}_{\Gamma}\left(E[n], E^{\prime}[n]\right) .
$$

In [18] (Prop. 4.2 and Example A3) the authors constructed infinitely many pairs of non-isogenous elliptic curves $E, E^{\prime}$ such that $\operatorname{Hom}_{\Gamma}\left(E[\ell], E^{\prime}[\ell]\right)=0$ for any odd prime $\ell$. Then $\operatorname{Br}(X) / \operatorname{Br}_{1}(X)$ is a finite abelian 2-group, so that only the archimedean place, 2 and the primes of bad reduction of $E$ and $E^{\prime}$ can turn up in the description of the Brauer-Manin set of $X$.

\section{Transcendental Brauer group and the Tate conjecture FOR DIVISORS}

In connection with Lemma 1.1 let us briefly discuss the following question (see also [17).

Question 2. Let $X$ be a smooth, proper and geometrically integral variety over a field $k$ finitely generated over $\mathbb{Q}$. We have the inclusion of groups $\operatorname{Br}(X) / \operatorname{Br}_{1}(X) \subset$ $\operatorname{Br}(\bar{X})^{\Gamma}$. Are these two groups finite?

It is well known that the finiteness of $\operatorname{Br}(\bar{X})^{\Gamma}$ is related to the Tate conjecture for divisors. This conjecture says that if $k$ is a field finitely generated over $\mathbb{Q}$, then for any prime $\ell$ the natural inclusion

$$
\left(\mathrm{NS}(\bar{X}) \otimes_{\mathbb{Z}} \mathbb{Q}_{\ell}\right)^{\Gamma} \hookrightarrow \mathrm{H}_{\text {ét }}^{2}\left(\bar{X}, \mathbb{Q}_{\ell}(1)\right)^{\Gamma}
$$

should be an isomorphism. The following partial answer to Question 2 must have been known to many people. It was noticed by one of us ten years ago.

Proposition 4.1. Let $X$ be a smooth, proper and geometrically integral variety over a field $k$ finitely generated over $\mathbb{Q}$. Assume the $\ell$-adic Tate conjecture for divisors.

(i) If $\operatorname{dim} X=2$, then $\operatorname{Br}(\bar{X})^{\Gamma}\{\ell\}$ is finite.

(ii) For $X$ of any dimension assume in addition the semisimplicity of the continuous $\Gamma$-module $\mathrm{H}_{e ̂ t}^{2}\left(\bar{X}, \mathbb{Q}_{\ell}(1)\right)$.

Then $\operatorname{Br}(\bar{X})^{\Gamma}\{\ell\}$ is finite.

Proof. Let $T_{\ell}(\operatorname{Br}(\bar{X}))$ be the $\ell$-adic Tate module of $\operatorname{Br}(\bar{X})$, and let $V_{\ell}(\operatorname{Br}(\bar{X}))=$ $T_{\ell}(\operatorname{Br}(\bar{X})) \otimes_{\mathbb{Z}_{\ell}} \mathbb{Q}_{\ell}$. The Kummer sequence gives a well-known exact sequence of continuous $\Gamma$-modules

$$
0 \longrightarrow \mathrm{NS}(\bar{X}) \otimes_{\mathbb{Z}} \mathbb{Q}_{\ell} \longrightarrow \mathrm{H}_{\text {ét }}^{2}\left(\bar{X}, \mathbb{Q}_{\ell}(1)\right) \longrightarrow V_{\ell}(\operatorname{Br}(\bar{X})) \longrightarrow 0 .
$$

When $X$ is a surface, the cup-product defines a non-degenerate Galois-equivariant bilinear pairing on $\mathrm{H}_{\text {ét }}^{2}\left(\bar{X}, \mathbb{Q}_{\ell}(1)\right)$ with values in $\mathbb{Q}_{\ell}$. The restriction of this pairing to $\mathrm{NS}(\bar{X}) \otimes_{\mathbb{Z}} \mathbb{Q}_{\ell}$ is non-degenerate; hence we obtain a direct sum decomposition of $\Gamma$-modules

$$
\mathrm{H}_{\text {ét }}^{2}\left(\bar{X}, \mathbb{Q}_{\ell}(1)\right) \cong\left(\mathrm{NS}(\bar{X}) \otimes_{\mathbb{Z}} \mathbb{Q}_{\ell}\right) \oplus V_{\ell}(\operatorname{Br}(\bar{X})) .
$$

We have the same conclusion under the semisimplicity hypothesis of part (ii). Now Tate's conjecture implies $V_{\ell}(\operatorname{Br}(\bar{X}))^{\Gamma}=0$.

For any abelian group $A$, the Tate module $T_{\ell}(A)$ is a torsion-free $\mathbb{Z}_{\ell}$-module. If $A$ is a torsion group whose $\ell$-primary component is of cofinite type, $T_{\ell}(A) \otimes_{\mathbb{Z}_{\ell}} \mathbb{Q}_{\ell} / \mathbb{Z}_{\ell}$ is the maximal $\ell$-divisible subgroup of $A$. We thus have an exact sequence

$$
0 \longrightarrow T_{\ell}(\operatorname{Br}(\bar{X})) \longrightarrow V_{\ell}(\operatorname{Br}(\bar{X})) \longrightarrow T_{\ell}(\operatorname{Br}(\bar{X})) \otimes_{\mathbb{Z}_{\ell}} \mathbb{Q}_{\ell} / \mathbb{Z}_{\ell} \longrightarrow 0
$$


where $T_{\ell}(\operatorname{Br}(\bar{X})) \otimes_{\mathbb{Z}_{\ell}} \mathbb{Q}_{\ell} / \mathbb{Z}_{\ell}$ is the maximal divisible subgroup $\operatorname{Br}(\bar{X})\{\ell\}_{\text {div }}$ of $\operatorname{Br}(\bar{X})\{\ell\}$. The exact sequence of Galois cohomology attached to (99) gives an exact sequence:

$$
V_{\ell}(\operatorname{Br}(\bar{X}))^{\Gamma} \longrightarrow\left(\operatorname{Br}(\bar{X})\{\ell\}_{\text {div }}\right)^{\Gamma} \longrightarrow \mathrm{H}^{1}\left(\Gamma, T_{\ell}(\operatorname{Br}(\bar{X}))\right) .
$$

By a general result of [20, Prop. 2.3] the kernel of the last arrow in (10) is the maximal divisible subgroup of $\left(\operatorname{Br}(\bar{X})\{\ell\}_{\text {div }}\right)^{\Gamma}$. From $V_{\ell}(\operatorname{Br}(\bar{X}))^{\Gamma}=0$ we conclude that the maximal divisible subgroup of $\left(\operatorname{Br}(\bar{X})\{\ell\}_{\text {div }}\right)^{\Gamma}$ is zero. Since $\left(\operatorname{Br}(\bar{X})\{\ell\}_{\text {div }}\right)^{\Gamma}$ is a subgroup of $\operatorname{Br}(\bar{X})\{\ell\}$, which is a torsion group of finite cotype, we see that $\left(\operatorname{Br}(\bar{X})\{\ell\}_{\text {div }}\right)^{\Gamma}$ is also a torsion group of finite cotype. It follows that $\left(\operatorname{Br}(\bar{X})\{\ell\}_{\text {div }}\right)^{\Gamma}$ is finite.

As already recalled, by [8], III (8.9), we have an exact sequence of continuous $\Gamma$-modules

$$
0 \longrightarrow \operatorname{Br}(\bar{X})\{\ell\}_{\text {div }} \longrightarrow \operatorname{Br}(\bar{X})\{\ell\} \longrightarrow \mathrm{H}_{\text {ét }}^{3}\left(\bar{X}, \mathbb{Z}_{\ell}(1)\right)_{\text {tors }} \longrightarrow 0 .
$$

The attached exact sequence of Galois cohomology gives an exact sequence

$$
0 \longrightarrow\left(\operatorname{Br}(\bar{X})\{\ell\}_{\text {div }}\right)^{\Gamma} \longrightarrow \operatorname{Br}(\bar{X})\{\ell\}^{\Gamma} \longrightarrow \mathrm{H}_{\text {ét }}^{3}\left(\bar{X}, \mathbb{Z}_{\ell}(1)\right)_{\text {tors }}^{\Gamma}
$$

Since $\mathrm{H}_{\text {ét }}^{3}\left(\bar{X}, \mathbb{Z}_{\ell}(1)\right)_{\text {tors }}$ is finite, our statement follows.

Remarks. 1. When $\mathrm{H}^{2}\left(X, O_{X}\right)=0$ the group $\operatorname{Br}(\bar{X})$ is finite, so Question 2 is trivial.

2. For abelian varieties or $K 3$ surfaces, the Tate conjecture for divisors is known. Here one can do better than Proposition 4.1] by the main theorem of [17, Question 2 has a positive answer in these cases.

\section{REFERENCES}

[1] M. Bright, Efficient evaluation of the Brauer-Manin obstruction. Math. Proc. Cambridge Philos. Soc. 142 (2007) 13-23. MR2296387 (2007k:14026)

[2] M. Bright, Evaluating Azumaya algebras on cubic surfaces. Manuscripta Math. 134 (2011) 405-421. MR2765718 (2012c:11136)

[3] J.-L. Colliot-Thélène et J.-J. Sansuc, La descente sur les variétés rationnelles, II, Duke Math. J. 54 (1987) 375-492. MR899402 (89f:11082)

[4] O. Debarre, Higher-Dimensional Algebraic Geometry, Universitext, Springer-Verlag, 2001. MR:1841091 (2002g:14001)

[5] B. Fantechi, L. Göttsche, L. Illusie, S.L. Kleiman, N. Nitsure, and A. Vistoli, Fundamental algebraic geometry. Grothendieck's FGA explained. Mathematical Surveys and Monographs 123, AMS, Providence, RI, 2005. MR2222646 (2007f:14001)

[6] K. Fujiwara, A proof of the absolute purity conjecture (after Gabber). In: Algebraic geometry 2000, Azumino, Adv. Stud. Pure Math. 36 (2002), pp. 153-183. MR.1971516 (2004d:14015)

[7] O. Gabber, Some theorems on Azumaya algebras. In: The Brauer group (Séminaire, Les Plans-sur-Bex, 1980) Lecture Notes in Math. 844, Springer-Verlag, Berlin-New York, 1981, pp. 129-209. MR611868 (83d:13004)

[8] A. Grothendieck, Le groupe de Brauer, I, II, III. In: Dix Exposés sur la Cohomologie des Schémas, North-Holland, Amsterdam, 1968, pp. 46-188. MR0244269 (39:5586a)

[9] E. Ieronymou, A.N. Skorobogatov and Yu.G. Zarhin, On the Brauer group of diagonal quartic surfaces. J. London Math. Soc. (2) 83 (2011), 659-672. arXiv:0912.2865 MR.2802504

[10] K. Kato, A Hasse principle for two-dimensional global fields. J. reine angew. Math. 366 (1986) 142-181. MR833016 (88b:11036)

[11] N. Katz, Applications of the weak Lefschetz theorem. Appendix to B. Poonen and J.F. Voloch, Random Diophantine equations. Progr. Math. 226 Arithmetic of higher-dimensional algebraic varieties (Palo Alto, CA, 2002), Birkhäuser, Boston, MA, 2004, pp. 175-184. MR2029869 (2005g:11055) 
[12] J. S. Milne, Arithmetic duality theorems, Persp. Math. 1, Academic Press, 1986. MR881804 (88e:14028)

[13] J.S. Milne, Étale cohomology. Princeton University Press, 1980. MR559531 (81j:14002)

[14] J-P. Serre, Corps locaux. Hermann, Paris, 1968. MR0354618 (50:7096)

[15] A.N. Skorobogatov, Descent on fibrations over the projective line. Amer. J. Math. 118 (1996) 905-923. MR1408492 (97k:11099)

[16] A. Skorobogatov, Torsors and rational points. Cambridge University Press, 2001. MR,1845760 (2002d:14032)

[17] A.N. Skorobogatov and Yu.G. Zarhin, A finiteness theorem for the Brauer group of abelian varieties and K3 surfaces. J. Alg. Geom. 17 (2008) 481-502. MR2395136 (2009d:14016)

[18] A.N. Skorobogatov and Yu.G. Zarhin, The Brauer group of Kummer surfaces and torsion of elliptic curves. J. reine angew. Math. 666 (2012) 115-140.

[19] Sir Peter Swinnerton-Dyer, Density of rational points on certain surfaces. Preprint, 2010.

[20] J. Tate, Relations between $K_{2}$ and Galois cohomology, Invent. Math. 36 (1976) 257-274. MR0429837 (55:2847)

CNRS, UMR 8628, Mathématiques, BÂtiment 425, Université Paris-Sud, F-91405 OrSAY, France

E-mail address: jlct@math.u-psud.fr

Department of Mathematics, South Kensington Campus, Imperial College London, SW7 2BZ England, United Kingdom - And - Institute for the Information Transmission Problems, Russian Academy of Sciences, 19 Bolshoi Karetnyi, Moscow, 127994 Russia

E-mail address: a.skorobogatov@imperial.ac.uk 